BULLETIN OF THE

AMERICAN MATHEMATICAL SOCIETY

Volume 79, Number 4, July 1973

\title{
MODULES OVER DIFFERENTIAL POLYNOMIAL RINGS
}

\author{
BY RICHARD E. BLOCK ${ }^{1}$ \\ Communicated by Alex Rosenberg, December 18, 1972
}

This note announces a number of results on the structure of differential modules over differential rings, where differential ring means a ring with a family of derivations and differential module means a module having a family of operators compatible with the derivations of the ring. To fix notation, throughout the paper we let $A$ denote an associative ring, $M={ }_{A} M$ an $A$-module, $\lambda$ the corresponding representation of $A$ in $M$, and $D=\left\{d_{i}\right\}_{i \in I}$ a family of derivations of $A$ (to $A$ ). We are concerned with the structure of $M$ when $M$ is $D$-admissible where we call $M$ $D$-admissible if the representation $\lambda$ can be extended to a representation $\rho$ of $A[Y ; D]$ in $M$. Here $A[Y ; D]$ denotes the ring of differential polynomials in the (noncommuting) indeterminates $Y=\left\{y_{i}\right\}_{i \in I}$; thus $A[Y ; D]$ is the free (left) module over $A$ with basis the free monoid on $Y$, with multiplication determined by the relations $\left\{y_{i} a-a y_{i}=d_{i} a \mid a \in A, i \in I\right\}$. For example, it can be shown that every projective $A$-module is $D$ admissible.

The main theorems announced here concern those $D$-admissible $M$ for which the representation $\rho$ of $A[Y ; D]$ in $M$ can be chosen to be irreducible; such $M$ will be completely determined under any of several finiteness conditions on $A$ or on $M$. The results are new even in the finite-dimensional case. While the results are about associative rings, they are in part motivated by the theory of Lie algebras. In fact, the main theorems can be regarded as generalizations of some classical results on Lie algebras. While these classical results fail hopelessly at prime characteristic, we shall see that associative versions of them do hold in a sharp form at characteristic $p$.

Differentiably irreducible (d.i.) modules. Recall that an additive mapping $t$ of $M$ to $M$ is called a differential transformation of $M$ if there is a mapping $d$ of $A$ to $A$ such that $t a-a t=\lambda(d a)$ for all $a$ in $A$; we then say that $t$ is a differential transformation of $M$ with respect to $d$. If $M$ is faithful then $d$ is necessarily a derivation of $A$. If for each $i$ in the index set $I$ there is a differential transformation $t_{i}$ with respect to the given derivation $d_{i}$, then $M$ is $D$-admissible, and conversely (take $\rho\left(y_{i}\right)=t_{i}$ ).

AMS (MOS) subject classifications (1970). Primary 16A72, 16A64, 16A48; Secondary 16A40, 16A46, 12H05, 17B99.

${ }^{1}$ Research supported in part by National Science Foundation Grant GP-23998. 
We call $M T$-irreducible for a set $T$ of differential transformations of $M$ if $A M \neq 0$ and $M$ has no proper submodule $N$ with $T N \subseteq N$. Also we call $M$ differentiably irreducible (d.i.) if $M$ is $T$-irreducible for some $T$ and hence for the set of all differential transformations of $M$. We remark that if $M$ is d.i. then it has characteristic 0 or a prime, as will $A$ if $M$ is faithful.

We now give some examples of d.i. modules which under suitable hypotheses will turn out to be a complete list. Since the set of differential transformations of $M$ contains the centralizer $\operatorname{Hom}_{A}(M, M)$, any direct sum of isomorphic irreducible $A$-modules is d.i. Another type of example is the regular module of the (commutative) $p$-truncated polynomial algebra $F\left[X_{1}, \ldots, X_{n}\right] /\left(X_{1}^{p}, \ldots, X_{n}^{p}\right)$ (for short we denote this by $\left.F_{[n]}\right)$ over a field $F$ of characteristic $p$. We combine these two types of examples by taking their tensor product. Thus let $S$ be a simple ring of characteristic $p$, and let $A$ be the truncated polynomial ring $S\left[X_{1}, \ldots, X_{n}\right] /\left(X_{1}^{p}, \ldots, X_{n}^{p}\right)$ $\left(=S \otimes_{\mathbf{z}_{p}} Z_{p[n]}=S_{[n]}\right)$ with coefficients in $S$. Also let $V$ be any direct sum of isomorphic irreducible $S$-modules, and let $M$ be the $A$-module $V\left[X_{1}, \ldots, X_{n}\right] /\left(X_{1}^{p}, \ldots, X_{n}^{p}\right)\left(=V \otimes_{\mathbf{Z}_{p}} Z_{p[n]}=V_{[n]}\right)$. Here $Z_{p}$ may be replaced by any field over which $S$ is an algebra without changing the ring $S_{[n]}$ or module $V_{[n]}$; in fact $S_{[n]}$ is also the group ring with coefficients in $S$ of the abelian group of type $(p, \ldots, p)$ ( $n$ times). We shall also write $S_{[0]}=S$ and $V_{[0]}=V$. In any case, $V_{[n]}$ is a faithful d.i. $S_{[n]}$-module. If $S$ (and so also $S_{[n]}$ ) is artinian then $V_{[n]}$ can also be described as a direct sum of reduced regular modules [4] of $S_{[n]}$. For convenience, if there is a subring $S$ of $A$ and an isomorphism of $S_{[n]}$ onto $A$ fixing every element of $S$ then we shall simply write $A=S_{[n]}$, and similarly for the modules $V_{[n]}$. The importance for differential ring theory of the rings $S_{[n]}$ is indicated by the main theorem of [2]: If a ring $B$ is differentiably simple (that is, $B^{2}=0$ and no proper ideal of $B$ is invariant under all derivations of $B$ ) and has a minimal (two-sided) ideal then either $B$ is simple or of the form $S_{[n]}$ for some simple subring $S$ of prime characteristic (and conversely).

THEOREM 1. Suppose that $A$ is left or right artinian and $M$ is faithful and d.i. Then $A$ is artinian, and either $A$ is simple or $A$ has a simple artinian subring $S$ of prime characteristic $p$ and ${ }_{s} M$ has a unital submodule $V$ such that $A=S_{[n]}$ and $M=V_{[n]}$ for some $n>0$. Thus $M$ is a direct sum of copies of the reduced regular module of $A$ (and conversely every such module over such an $A$ is faithful and d.i.). ${ }^{2}$

The proof uses the author's theorems [2] on differentiably simple and semisimple rings and theorems on quasi-Frobenius rings [4]. The first step in the proof shows that $A$ is differentiably simple.

THEOREM 2. Suppose in Theorem 1 that $A$ and $M$ are finite dimensional

\footnotetext{
${ }^{2} \mathrm{~A}$ preliminary version of this result and that of Corollary 2 below was given in [3].
} 
over an algebraically closed field $F$ and that $M$ is T-irreducible where $T$ is a set of commuting F-linear differential transformations. Then $V$ (or $M$ in case $A$ is simple) is irreducible.

Henceforth let $C$ denote the centralizer $\operatorname{Hom}_{A}(M, M)$ (and $C_{C}$ and ${ }_{C} C$ its right and left regular modules).

Theorem 3 (Generalized Schur's Lemma). Suppose that $M$ is d.i., finitely generated, and has a minimal submodule. Then $M, C_{C}$ and ${ }_{C} C$ all have the same finite composition length.

The proof of Theorem 3 is of course considerably longer than that of the classical Schur's lemma, which can be stated as follows: If $M$ has length one then so does $C_{C}$ (and ${ }_{C} C$ ).

THEOREM 4. Let $M$ be d.i., finitely generated and have a minimal submodule. (i) If $A$ has characteristic 0 then $M$ is a finite direct sum of isomorphic copies of an irreducible A-module. (ii) If $A$ has prime characteristic suppose in addition that $M$ is faithful, $A$ has a unit, $A$ is differentiably simple with a minimal ideal, and $A$ contains the center $z C$ of $C$; then there is a simple subring $S$ of $A$ and a submodule ${ }_{s} V$ of ${ }_{S} M$ which is a finite direct sum of isomorphic copies of an irreducible $S$-module such that $A=S_{[n]}$ and $M=V_{[n]}$ for some $n \geqq 0$; whether or not $A$ contains $z C$, the same conclusions hold for $A C$ in place of $A$.

The proof involves an extension of some of the methods used in the proof of the author's theorem [2] on differentiably simple rings. The hypothesis that $A$ contains $z C$ holds if ${ }_{A} M$ has the double centralizer property. The hypothesis cannot be deleted even if $A$ is simple, as is shown by the following result.

THEOREM 5. At each prime characteristic there is a simple ring with unit which has a faithful unital module of finite composition length which is not completely reducible but is d.i., in fact $\{t\}$-irreducible for some differential transformation $t$.

The author has other results on d.i. modules which involve a completely different approach from that of the results above. These results are related to work of Blattner [1] and Dixmier [5], and are being announced separately.

Connections with representations of Lie algebras. Let $L$ be a Lie ring, $K$ an ideal of $L, M$ an $L$-module with corresponding representation $\mu$, and $A$ the enveloping ring of ${ }_{K} M$ (that is, the associative subring of $\operatorname{Hom}_{\mathbf{z}}(M, M)$ generated by $\left.\{\mu(y) \mid y \in K\}\right)$. Then $L$ gives a family $D=D_{L}$ of derivations of $A$ via $d_{x} a=[\mu(x), a](x \in L, a \in A)$, and ${ }_{A} M$ is faithful 
and $D$-admissible, with $T=\{\mu(x) \mid x \in L\}$ a family of differential transformations with respect to $D$. Moreover ${ }_{A} M$ is $T$-irreducible if and only if ${ }_{L} M$ is irreducible.

Thus if $L$ has characteristic 0 and ${ }_{L} M$ is irreducible, and if either the enveloping ring of ${ }_{K} M$ is (left or right) artinian or ${ }_{K} M$ is finitely generated with a minimal submodule, then Theorem 1 or Theorem 4(i) implies that ${ }_{K} M$ is a direct sum of isomorphic irreducible submodules. In the finitedimensional characteristic 0 case this result implies Lie's theorem (that an irreducible solvable Lie algebra of linear transformation is commutative), by induction on the dimension of $L$; it then implies the key part of the Cartan-Jacobson theorem (namely that the radical of a completely reducible Lie algebra of linear transformations equals the center). This provides a proof of these classical results not involving any trace argument.

In the case of prime characteristic, Theorems 1 and 2 above lead to a generalization of Zassenhaus' results [6] on Lie algebra representations which are irreducible with respect to a single differential transformation. For example, one immediately gets the following.

COROLlary 1. An irreducible representation of a finite-dimensional solvable Lie algebra over an algebraically closed field of characteristic $p$ has degree a power of $p$.

Our theorems above are of use in the problem of determining the simple Lie algebras of prime characteristic. Another nonassociative application gives a structure theorem for the irreducible representations of flexible algebras (including noncommutative Jordan algebras).

Characterizations of $D$-semisimple artinian rings. Henceforth we shall assume that the ring $A$ is artinian with unit and that all modules considered are unital. We recall [2] that the $D$-radical of $A$ is defined to be the largest $D$-invariant radical ideal of $A$, and that $A$ is called $D$-semisimple if its $D$-radical is 0 . Further [2], $A$ is $D$-semisimple if and only if $A$ is a direct sum of ideals which are either simple or of the form $S_{[n]}$.

We shall say that the $A$-module $M$ is $D$-irreducibly admissible if the representation of $A$ in $M$ admits an extension to a representation of $A[Y ; D]$ in $M$ which is irreducible; in particular then $M$ is d.i. Our final results show that some classical theorems on modules have analogues in our situation.

THEOREM 6. The following properties of the A-module $M$ are equivalent: (i) $M$ is a direct sum of minimal $D$-admissible submodules; (ii) $M$ is a direct sum of D-irreducibly admissible submodules; (iii) Every D-admissible submodule of $M$ has a complement in $M$ and $M$ is $D$-admissible; (iv) $A /(0: M)$ is D-semisimple. 
THEOREM 7. The following are equivalent: (i) $A$ is D-semisimple; (ii) Every D-admissible A-module satisfies the properties of Theorem 6; (iii) ${ }_{A} A$ satisfies the properties of Theorem 6; (iv) Every D-admissible $A$ module is projective; (v) Every D-admissible A-module is injective.

Corollary 2. Let $A$ be $D$-semisimple. Then $M$ is $D$-admissible if and only if $M$ is a direct sum of modules each of which is a reduced regular module (and thus of the form $V_{[n]}, V$ irreducible) for one of the D-simple summands of $A$ (of the form $S_{[n]}$, with $n=0$ if $S$ has characteristic 0 ) and is annihilated by the remaining $D$-simple summands of $A$.

\section{REFERENCES}

1. R. J. Blattner, Induced and produced representations of Lie algebras, Trans. Amer. Math. Soc. 144 (1969), 457-474.

2. R. E. Block, Determination of the differentiably simple rings with minimal ideal, Ann. of Math. (2) 90 (1969), 433-459. MR 40 \# 4319.

3. - Modules sur les anneaux de polynômes différentiels, Université de Paris, Faculté des Sciences d'Orsay, Séminaire d'Algèbre Non Commutative 1970-71, 3.1-3.10.

4. C. W. Curtis and I. Reiner, Representation theory of finite groups and associative algebras, Pure and Appl. Math., Vol. II, Interscience, Wiley, New York, 1962. MR 26 \# 2519.

5. J. Dixmier, Sur les représentations induites des algèbres de Lie, J. Math. Pures Appl. 50 (1971), 1-24.

6. H. Zassenhaus, Über Lie'sche Ringe mit Primzahlcharakteristik, Abh. Math. Sem. Hamburg 13 (1939), 1-100.

Department of Mathematics, University of CAlifornia, Riverside, California 92502 